\title{
ASYMPTOTIC DISTRIBUTION OF THE ZEROS OF CERTAIN LAGRANGE INTERPOLANTS
}

\author{
by M. A. BOKHARI and M. IQBAL
}

(Received 4th May 1993)

\begin{abstract}
We consider rational functions of the form $f_{m}(z)=z^{m} /(z-\rho)$ which are analytic in $|z|\langle\rho, \rho>1$, and establish that the asymptotic distribution of the zeros of their Taylor sections and Lagrange interpolants at uniformly distributed nodes is similar. This notion is also illustrated computationally. We conjecture that a similar result can be expected for any function analytic in $|z|<\rho$.
\end{abstract}

1991 Mathematics subject classification: 30C15, 41A05.

\section{Introduction}

An elegant result of J. L. Walsh ([12, p. 153]) provides a close relationship between the partial sections of the Taylor series of an analytic function and its Lagrange interpolants in the roots of unity. During the last decade many mathematicians extended this result in various directions $([1]-[4],[10])$. On the other hand, the problem related to the distribution of zeros of the sections of power series of an analytic function has a long and respectable history ([5], [8], [11]). At this point it seems worthwhile to question whether something can be said about the asymptotic distribution of the zeros of Lagrange interpolants to $f$ on uniformly distributed nodes in the complex plane.

Let $A_{\rho}$ be the class of functions $f$ analytic in the open disk $|z|<\rho$ but non-analytic on $|z|=\rho$. For $f(z)=\sum_{k=0}^{\infty} a_{k} z^{k} \in A_{\rho}$, let $S_{n}(z, f)=\sum_{k=0}^{n} a_{k} z^{k}$, and let $L_{n}^{\sigma}(z, f)$ denote the polynomial of degree $n$ which interpolates $f$ in the $n+1$ roots of $z^{n+1}-\sigma^{n+1}=0$ where $0<\sigma<\rho$. Now a simple but interesting situation provides us a further motivation to discuss the foregoing question:

Example 1.1. Select $f_{0}(z)=(\rho-z)^{-1}$ with $\rho>\sigma>0$. Then it is easy to see that

$$
\begin{gathered}
S_{n}\left(z, f_{0}\right)=\frac{\rho^{n+1}-z^{n+1}}{\rho^{n+1}(\rho-z)} \\
L_{n}^{\sigma}\left(z, f_{0}\right)=\frac{\rho^{n+1}}{\rho^{n+1}-\sigma^{n+1}} S_{n}\left(z, f_{0}\right)
\end{gathered}
$$

and 


$$
L_{n}\left(z, f_{0}\right)-S_{n}\left(z, f_{0}\right)=\frac{\sigma^{n+1}}{\rho^{n+1}-\sigma^{n+1}} S_{n}\left(z, f_{0}\right)
$$

This shows that the $n$ zeros of $S_{n}\left(z, f_{0}\right)$, which are uniformly distributed on the circle $|z|=\rho$ (except for $z=\rho$ ), are identical to those of $L_{n}^{\sigma}\left(z, f_{0}\right)$ and $L_{n}^{a}\left(z, f_{0}\right)-S_{n}\left(z, f_{0}\right)$.

Instead of $f_{0}(z)$, if our choice is slightly modified to $f_{1}(z)=z(\rho-z)^{-1}$, then

$$
S_{n}\left(z, f_{1}\right)=\frac{z\left(\rho^{n}-z^{n}\right)}{\rho^{n}(\rho-z)},
$$

and

$$
L_{n}^{\sigma}\left(z, f_{1}\right)=\frac{1}{\rho^{n+1}-\sigma^{n+1}}\left[\rho^{n+1} S_{n}\left(z, f_{1}\right)+\sigma^{n+1}\right]
$$

Contrary to the outcome of the Example 1.1, we note that none of the zeros of $S_{n}\left(z, f_{1}\right)$ is identical to a zero of $L_{n}^{\sigma}\left(z, f_{1}\right)$. In fact, $S_{n}\left(z, f_{1}\right)$ possesses one of the zeros at the origin and the rest are uniformly distributed on $|z|=\rho$. On the other hand the location of the zeros of $L_{n}^{\sigma}\left(z, f_{1}\right)$ is not obvious from its expression (1.1).

The aim of the present note is to investigate the asymptotic behaviour of the zeros of $L_{n}^{\sigma}\left(z, f_{m}\right)$ where $m$ is a fixed nonnegative integer and

$$
f_{m}(z)=z^{m} /(\rho-z)
$$

We show that asymptotically the distribution of the zeros of $L_{n}^{\sigma}\left(z, f_{m}\right)$ and $S_{n}\left(z, f_{m}\right)$ is similar. This notion is also illustrated graphically by computing the zeros of the two polynomials. In the last section, we suggest a conjecture related to the rational function $\left(L_{n}^{o}(z, f)-S_{n}(z, f)\right) / S_{n}(z, f), f \in A_{\rho}, \rho>1$, which provides us an insight about the asymptotic distribution of the zeros of $L_{n}^{\sigma}(z, f)$.

\section{Notation and main result}

Let $m$ be a fixed non-negative integer. For any integer $n \geqq m$, we shall write

$$
n(m):=n-m+1 \text {. }
$$

Let

$$
\omega_{l, n(m)}:=\exp (2 l i \pi / n(m)), \quad l=1,2, \ldots, n(m)
$$

Since

$$
S_{n}\left(z, f_{m}\right)=\frac{z^{m}\left(\rho^{n(m)}-z^{n(m)}\right)}{\rho^{n(m)}(\rho-z)}
$$


we observe that $S_{n}\left(z, f_{m}\right)$ has a zero of multiplicity $m$ at $z_{0}=0$ and its remaining $n(m)-1$ zeros are given by

$$
z_{l, n(m)}=\rho \cdot \omega_{l, n(m)}, \quad l=1,2, \ldots, n(m)-1
$$

For each $l=1,2, \ldots, n(m)-1$, we set

$$
\varepsilon_{l, n(m)}=\frac{1}{2} \min \left(\min _{\substack{l \neq j \\ 1 \leqq j \leqq n(m)-1}}\left|z_{l, n(m)}-z_{j, n(m)}\right|, \frac{\rho^{2}}{\sigma}-\rho, \rho-1\right)
$$

where $\sigma>0$ and $\rho>\max \{1, \sigma\}$. If we denote by $O_{\varepsilon_{l, n(m)}}\left(z_{l, n(m)}\right)$ the open sphere with centre at $z_{l, n(m)}$ and radius $\varepsilon_{l, n(m)}$ then our main result can be stated as:

Theorem 2.1. Let $f_{m}(z)=z^{m} /(\rho-z)$ where $m$ is a fixed non-negative integer. Let $\sigma>0$ and $\rho>\max \{1, \sigma\}$. Then:

(A) For every sufficiently small neighbourhood $K$ of $z_{0}=0$, there exists a positive integer $N(K)$ such that $K$ contains exactly $m$ zeros of $L_{n}^{\sigma}\left(z, f_{m}\right), n \geqq N(K)$.

(B) For all sufficiently large $n$ and for all $l=1,2, \ldots, n(m)-1$, the open sphere $O_{\varepsilon_{1, n(m)}}\left(z_{l, n(m)}\right)(c f .(2.3),(2.4))$ contains exactly one zero of $L_{n}^{\sigma}\left(z, f_{m}\right)$.

The proof of Theorem 2.1 is partly based on the behaviour of the rational function $P_{n}\left(z, f_{m}\right) / S_{n}\left(z, f_{m}\right)$ where

$$
P_{n}\left(z, f_{m}\right):=L_{n}^{\sigma}\left(z, f_{m}\right)-S_{n}\left(z, f_{m}\right)
$$

More precisely, if $C_{l, n(m)}$ denotes the boundary of $O_{\varepsilon_{l, n(m)}}\left(z_{l, n(m)}\right), l=1,2, \ldots, n(m)-1$, we prove:

Lemma 2.1. With all the conditions specified in the hypothesis of Theorem 2.1, we have

$$
L_{n}^{\sigma}\left(z, f_{m}\right)=\frac{\rho^{m}\left(\rho^{n+1}-z^{n+1}\right)}{\left(\rho^{n+1}-\sigma^{n+1}\right)(\rho-z)}-\frac{\rho^{m}-z^{m}}{\rho-z}
$$

(ii) for all sufficiently large values of $n$

$$
\left|\frac{P_{n}\left(z, f_{m}\right)}{S_{n}\left(z, f_{m}\right)}\right|<1, \quad \forall z \in C_{l, n(m)}
$$

$$
l=1,2, \ldots, n(m)-1 .
$$

Proof. The formula (2.6) follows from the interpolatory properties of the polynomial $L_{n}^{\sigma}\left(z, f_{m}\right)$. 
In order to establish (2.7), first we note that

$$
P_{n}\left(z, f_{m}\right)=\frac{\sigma^{n+1}}{\rho^{n-m+1}\left(\rho^{n+1}-\sigma^{n+1}\right)} \cdot \frac{\rho^{n+1}-z^{n+1}}{\rho-z} .
$$

The choice of $\varepsilon_{l, n(m)}$ guarantees that the polynomial $S_{n}\left(z, f_{m}\right)$ does not possess any zero on the circle $C_{l, n(m)}$. Moreover,

$$
1 \leqq|z|<\frac{\rho^{2}}{\sigma}, \quad \forall z \in C_{l, n(m)}
$$

Since $\sigma<\rho$, a straightforward calculation from (2.8) and (2.2) now yields the estimate

$$
\frac{P_{m}\left(z, f_{m}\right)}{S_{n}\left(z, f_{m}\right)}=O\left(\frac{\sigma}{\rho}\right)^{n+1} \quad \forall z \in C_{l, n(m)}, \quad \text { as } \quad n \rightarrow \infty
$$

Thus (2.7) holds.

Proof of Theorem 2.1. Using the integral representation of $L_{n}^{\sigma}\left(z, f_{m}\right)$ and $f_{m}(z)$, we have

$$
L_{n}^{\sigma}\left(z, f_{m}\right)-f_{m}(z)=\frac{1}{2 \pi i} \int_{\Gamma} \frac{f_{m}(t)}{t-z} \cdot \frac{\sigma^{n+1}-z^{n+1}}{t^{n+1}-\sigma^{n+1}} d t, \quad|z|<\rho,
$$

where $\Gamma$ is the circle $|t|=R$ with $\sigma<R<\rho$. Consequently, the sequence of polynomials $L_{n}^{\sigma}\left(z, f_{m}\right)$ converges uniformly to $f_{m}(z)$ in every closed subregion of $|z|<\rho$. An application of Hurwitz theorem ([7]) now leads us to establish part (A) of Theorem 2.1.

For part (B), first note that

$$
L_{n}^{\sigma}\left(z, f_{m}\right)=P_{n}\left(z, f_{m}\right)+S_{n}\left(z, f_{m}\right)
$$

Because of the relation (2.7), Rouchés theorem [7] confirms that $L_{n}^{\sigma}\left(z, f_{m}\right)$ has the same number of zeros interior to $C_{l, n(m)}$ as does the polynomial $S_{n}\left(z, f_{m}\right)$. Since $S_{n}\left(z, f_{m}\right)$ has exactly one zero inside $C_{l, n(m)}$, this completes the proof of Theorem 2.1.

Remark 2.1. Since $\varepsilon_{l, n(m)} \rightarrow 0$ as $n \rightarrow \infty$, it follows from Theorem 2.1 that the asymptotic distribution of the zeros of $S_{n}\left(z, f_{m}\right)$ and $L_{n}^{\sigma}\left(z, f_{m}\right)$ is similar.

\section{Discussion of numerical results}

To illustrate our main result we have used the ZPOCC program in the IMSL library 
in order to list the zeros of the two polynomials $S_{n}\left(z, f_{m}\right)$ and $L_{n}^{a}\left(z, f_{m}\right)$. The location of these zeros around the circle $|z|=\rho$ is shown for various values of $n$ separately in the computer-generated figures explained below:

We select $\rho=6$ and $(\sigma, m)=(2,8)$. The three figures corresponding to $n=10,15,21$ show that the Taylor polynomial $S_{n}\left(z, f_{8}\right)$ has a zero of order 8 at $z_{0}=0$ and its remaining $(n-8)$ zeros are distributed on the circle $|z|=6$. The $n$ zeros of $S_{n}\left(z, f_{8}\right)$ and $L_{n}^{2}\left(z, f_{8}\right)$ are represented respectively by the symbols $\triangle$ and $O$ in the three figures. We notice that exactly eight of the $n$ zeros of $L_{n}^{2}\left(z, f_{8}\right)$ move toward the zero of $S_{n}\left(z, f_{8}\right)$ at $z_{0}=0$ as $n$ attains larger values whereas each one of its remaining $(n-8)$ zeros gets closer to the $n-8$ zeros of $S_{n}\left(z, f_{8}\right)$ lying on the circle $|z|=6$.

\section{Concluding remarks}

In general, if $f \in A_{\rho}, \sigma>0$, and $\rho>\max \{1, \sigma\}$ then for every $z \in C$ with $|z|<\rho$, it is well known that

and

$$
\left.\begin{array}{l}
\lim _{n \rightarrow \infty} S_{n}(z, f)=f(z) \\
\lim _{n \rightarrow \infty} L_{n}^{o}(z, f)=f(z)
\end{array}\right\}
$$

Since the convergence in (4.1) is uniform on any compact subset of the region $D_{\rho}=\{z \in C:|z|<\rho\}$, we have the following result which is an immediate consequence of Hurwitz theorem [7]:

Theorem 4.1. If $z_{0}$ is an $m$-fold zero of $f \in A_{\rho}$ in the region $D_{\rho}$ then for all sufficiently small $\varepsilon>0$, the disk $\left|z-z_{0}\right|<\varepsilon$ contains exactly $m$ zeros (counted with their multiplicities) of each of the polynomials $S_{n}(z, f)$ and $L_{n}^{\sigma}(z, f), n \geqq N(\varepsilon)$.

Remark 4.1. If $f \in A_{\rho}$ has $r$ zeros, say $z_{j},(j=1, \ldots, r)$, (counted with their multiplicities) in $D_{\rho}$ and if $\xi_{j, n}$ and $\eta_{j, n},(j=1, \ldots, r)$, are the respective zeros of $S_{n}(z, f)$ and $L_{n}^{o}(z, f)$ lying in the neighbourhood of $z_{j}$ as narrated in Theorem 4.1 , then

$$
\lim _{n \rightarrow \infty}\left|\xi_{j, n}-\eta_{j, n}\right|=0 . \quad j=1,2, \ldots, r
$$

Besides the $r$ zeros described in the above remark, we are also interested in discussing the asymptotic behaviour of the remaining zeros of $S_{n}(z, f)$ and $L_{n}^{\sigma}(z, f)$ lying in the region $D_{\rho}$. For this, first we note that

$$
P_{n}(z, f):=L_{n}^{o}(z, f)-S_{n}(z, f)
$$




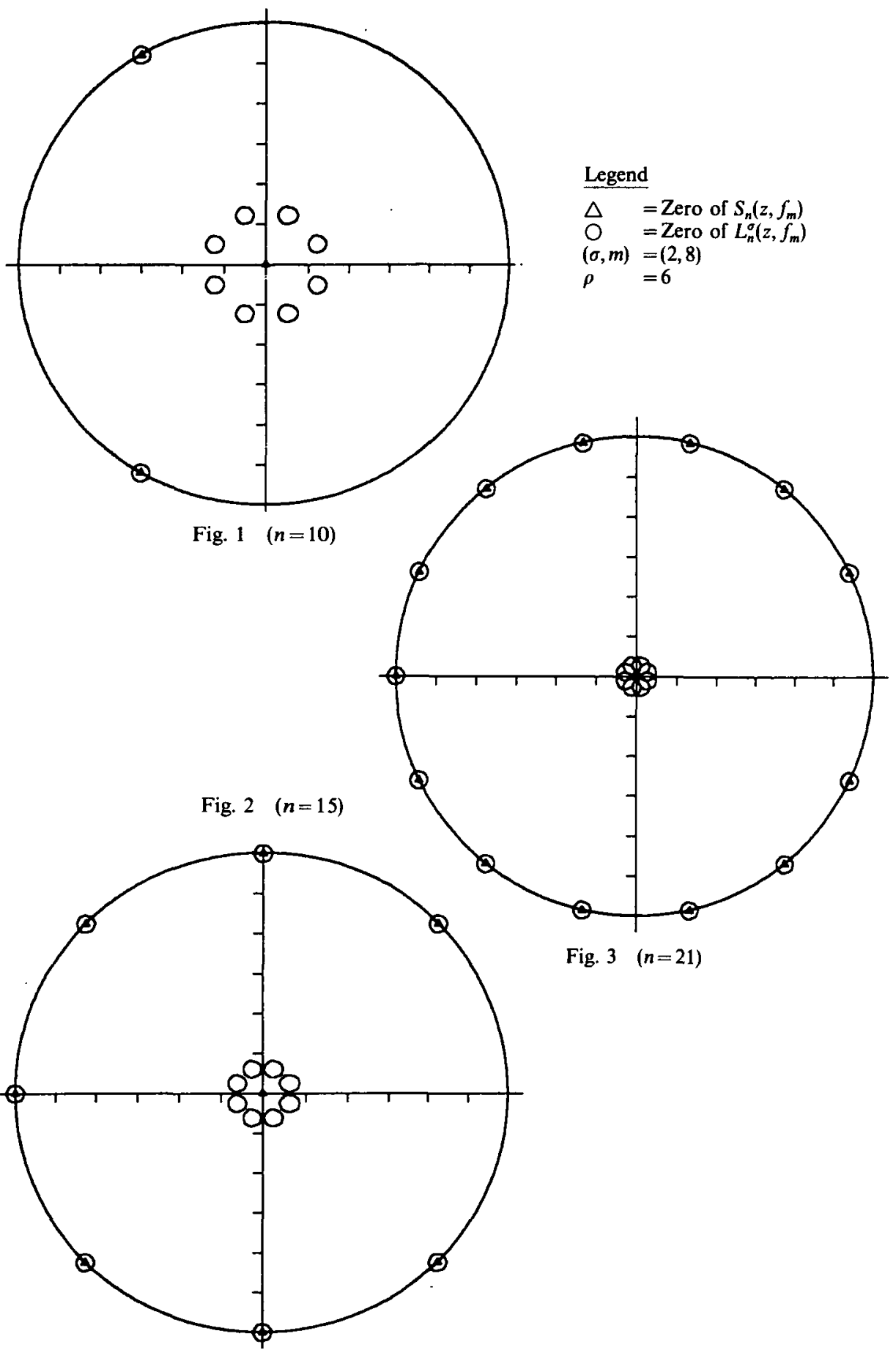




$$
\begin{aligned}
& =\frac{1}{2 \pi i} \int_{\Gamma} \frac{f(t)}{t-z} \cdot \frac{\sigma^{n+1}\left(t^{n+1}-z^{n+1}\right)}{t^{n+1}\left(t^{n+1}-\sigma^{n+1}\right)} d t \\
& =O\left(\left(\frac{|z| \sigma}{\rho^{2}}\right)^{n}\right)
\end{aligned}
$$

and

$$
\begin{aligned}
S_{n}(z, f) & =\frac{1}{2 \pi i} \int_{\Gamma} \frac{f(t)}{t-z} \cdot \frac{t^{n+1}-z^{n+1}}{t^{n+1}} d t \\
& =O\left(\max \left(1,\left(\frac{\lfloor z}{\rho}\right)^{n}\right)\right)
\end{aligned}
$$

where $\Gamma$ is the circle $|t|=R, \sigma<R<\rho$.

Next assume that $z_{1, n}, z_{2, n}, \ldots, z_{l, n}$ are the only distinct zeros of $S_{n}(z, f)$ in $D_{\rho}$ such that

$$
z_{k, n} \neq \xi_{j, n}, \quad k=1, \ldots, l \text { and } j=1, \ldots, r
$$

where $\xi_{j, n},(j=1, \ldots, r)$, are the zeros of $S_{n}(z, f)$ considered in Remark 4.1. Let

$$
\varepsilon_{k, n}=\frac{1}{2} \min \left\{\min _{\substack{1 \leq j \leq l \\ j \neq k}}\left|z_{j, n}-z_{k, n}\right|, \quad \rho-1, \quad \frac{\rho^{2}}{\sigma}-\rho\right\} .
$$

In the light of our Lemma 2.1 and the estimates (4.2) and (4.3), it seems appropriate to suggest the following:

Conjecture. Let $f \in A_{\rho}, \sigma>0$ and $\rho>\max \{1, \sigma\}$. If $C_{k, n}$ denotes the boundary of the disk $\left|z-z_{k, n}\right|<\varepsilon_{k, n}, k=1,2, \ldots, l$, then for all sufficiently large values of $n$

$$
\left|\frac{P_{n}(z, f)}{S_{n}(z, f)}\right|<1, \quad \forall z \in C_{k, n}
$$

$k=1,2, \ldots$, l. Moreover, $\varepsilon_{k, n} \rightarrow 0$ as $n \rightarrow \infty$.

Note that $S_{n}(z, f)$ has exactly one zero inside the circle $C_{k, n}$. Therefore, due to Rouché's theorem [7], $L_{n}^{\sigma}(2, f)$ will have exactly one zero, say $\tilde{z}_{k, n}$ on or within $C_{k, n}$, $(k=1, \ldots, l)$ if the above conjecture is valid. This will lead us to conclude that the asymptotic distribution of the zeros of $S_{n}(z, f)$ and $L_{n}^{o}(z, f)$ is similar.

As far as the location of the zeros of $S_{n}(z, f)$ is concerned when $f \in A_{\rho}$ and $1<\rho<\infty$, 
Jentzch [6] proved that for any $\varepsilon>0$, and for any $\phi(0 \leqq \phi \leqq 2 \pi)$, there are infinitely many values of $n$ having at least one zero in the disk

$$
\left|z-\rho e^{i \phi}\right|<\varepsilon
$$

Later on, Szegö [9] established the existence of an infinite sequence $\left\{n_{q}\right\}_{q=1}^{\infty}$ of positive increasing integers such that as $q \rightarrow \infty$, the arguments of the zeros of $S_{n_{q}}(z, f)$ are equidistributed in the sense of Weyl. We stress that the results of Jentzch and Szegö are valid for $L_{n}^{\sigma}(z, f), \sigma<\rho$, in place of $S_{n}(z, f)$ provided that our conjecture is true.

Acknowledgement. The authors acknowledge with thanks the research and computer facilities availed at King Fahd University of Petroleum and Minerals during the preparation of this paper.

\section{REFERENCES}

1. M. A. Bokhari, On certain sequences of least squares approximants, Bull. Austral. Math. Soc. 38 (1988), 415-422.

2. M. A. BoKharı, Equiconvergence of some sequences of complex interpolating rational functions, J. Approx. Th. 55 (1988), 205-219.

3. M. A. BoKHARI, Converse results in the theory of equiconvergence of interpolating rational functions, Rocky Mount. J. Math. 19 (1989), 73-81.

4. A. S. Cavaretta, A Sharma and R. S. Varga, Interpolation in the roots of unity: an extension of a theorem of J.L. Walsh, Resultate Math. 3 (1981), 155-191.

5. A. Edrei, E. B. SAfF and R. S. VArga, Zeros of Sections of Power Series (Lecture Notes in Mathematics 1002, Springer-Berlag, Berlin, 1983).

6. R. Jentzsch, Untersuchungen zur Theorie der Folgen analytischer Functionen, Acta Math. 41 (1917), 219-251.

7. M. Marden, Geometry of Polynomials (Mathematical Surveys No. 3, Amer. Math. Soc., Providence, RI, 1966).

8. P. C. Rosenbloom, Distribution of Zeros of Polynomials, in Lectures on Functions of a Complex Variable (W. Kaplan, ed., University of Michigan Press, Ann Arbor, 1955), 265-285.

9. G. Szego, Über die Nullstellen von Polynomen die in einem kreise gleichmässig konvergieren, Sitzungsber. Berl. Math. Ges. 21 (1922), 59-64.

10. R. S. VARGA, Topics in polynomial and rational interpolation and approximation chapter IV. Seminaire de Math. Superieures, Monreal, 1982).

11. R. S. VARGA, Scientific Computation on Mathematical Problems and Conjectures (CBMSNSF Regional Conference Series in Applied Math., SIAM, Philadelphia, 1990).

12. J. L. W $\mathrm{W}_{\mathrm{ALSH}}$, Interpolation and approximation by rational functions in the complex domain (A.M.S. Colloq. Publications, Vol. XX, Providence, R.I., 5th ed., 1969).

Department of Mathematical Sciences

King Fahd University of Petroleum and Minerals

Dhahran 31261, Saudi Arabia

E-Mail: FACL049@SAUPM00.BITNET. 